\title{
DECISION-MAKING IN TEAMWORKS: STICKY NOTES TOOL FOR DEGREE STUDENTS
}

\author{
Beatriz Andres, Raquel Sanchis, Raul Poler \\ Centro de Investigación en Gestión e Ingeniería de la Producción, Escuela Politécnica \\ Superior de Alcoy, Universitat Politècnica de València, Centre d'Innovació i Investigació \\ (Alicante) (SPAIN)
}

\begin{abstract}
This paper is focused on the presentation of an open-source online tool based on the methodology of sticky notes tools to support Industrial Engineering degree students in the practical work of decisionmaking and in the teamwork's practical sessions. The main aim of this paper is to identify a tool to support students, as future industrial engineers, in the decision-making process through teamwork, in the establishment of strategic policies, and in the process of creating solutions, amongst others. Moreover, three different case study are provided with the main objective of showing the potential of the proposed tool in the scope of decision-making in teamwork's.
\end{abstract}

Keywords: decision-making, brainstorming, design thinking, enterprise strategies, practical work, teamwork.

\section{INTRODUCTION AND MOTIVATION}

Considering the importance of the decision-making process and teamwork's in the Industrial Engineering degree, this paper focuses on the analysis of open-source tools to support Industrial Engineering degree students in the practical work of decision-making and in the teamwork's practical lessons. The analysed tools are based on online sticky notes canvas to support the decision making process and the teamwork skills.

The Universitat Politècnica de València (UPV) is currently involved in the incorporation of transversal skills into the curriculum of graduates. For the students, this is an opportunity to acquire transferable skills and accredit their training in these skills. While for the UPV, the inclusion of transversal skills is considered as valuable in terms of improvement qualifications, and national and international accreditations. UPV considers the importance of defining transversal competences in addition to the general and specific competences associated to each subject in each of the degrees offered by the university.

Thirteen are the transversal skills considered by the UPV. Initially, the entity responsible for the degree has established the relations between the degree and the thirteen transversal skills, considering the definition of each skill and the objectives pursued by each degree. Next, the academic committee of the degree has established checkpoints taking into account, on the one hand, the relation with the transversal skills set by the entity responsible for the degree and, on the other hand, the level of contribution of each subject at each skill defined in the degree-teaching guide. The UPV intends that, instead of each subject assesses independently each transversal skill, which has been initially defined as a control point, an holistic approach is adopted in the definition of subjects checkpoints, design subject for evaluation of transversal skills and evaluation of results.

The transversal competences related to the decision-making process, in which this paper focuses, are associated with the understanding and integration; practical application and thinking; analysis and problems resolution; creativity and entrepreneurship; teamwork and leadership; effective communication; critical thinking, and planning and time management.

\subsection{Transversal competences in the decision-making process}

Graduates and employers do not hesitate to emphasize the great importance that transversal and generic skills have in the professional performance of graduates. As a result, new degrees and postgraduate titles explicitly incorporate the requirement that students have to train in transversal skills, evaluating their level of achievement. An overview of each of the traversal skills, related with the decision-making process, is provided next: 
Understanding and integration. Understanding means, "to perceive and have a clear idea of what is said or done, discovering something in a deep sense." To show that something has been understood, the person identifies and retrieves information and explains it in its own words, interpreting and integrating ideas from its own perspective.

Practical application and thinking. The student, in his/her real life, needs to be prepared to deal with situations where applying recipes or formulas is not enough and where decisions or proposed solutions must be argued and adjusted to available resources. In this sense, this skill develops the mind-set led to action, which allows to adapt to new situations, make decisions and, consequently, act.

Analysis and Troubleshooting. The problems are new situations that require individuals to respond with new behaviours. Solving a problem involves performing tasks that require processes with more or less complex reasoning and, in many cases, it is not simply an associative and routine action. The aim of this transversal skill is, therefore, that the student is able to apply structured problem-solving procedures, thereby promoting their ability to learn, understand and apply knowledge autonomously.

Innovation, creativity and entrepreneurship. Innovation is understood as the ability to provide satisfactory answers to the personal, organizational and social needs, modifying processes and/or results to generate new value response. In turn, the development of this skill requires both thinking in a different manner to provide different perspectives (creativity), and the commitment of certain resources on his/her own initiative, in order to explore an opportunity, assuming the risk that this entails (venture).

Teamwork and leadership. Teamwork involves creating and developing a climate of mutual trust between components that allows working responsibly and cooperatively. The most appropriate term to describe this situation is "sharing": sharing knowledge, commitment and responsibility. It involves the distribution of tasks and roles and the respect of the norms and rules established by and for the group.

Effective communication. Communicate effectively means having developed the ability to transmit knowledge and express ideas and arguments in a clear, rigorous and convincing way, both orally and in writing, using the appropriate resources properly and adapting to the circumstances and the type of audience.

Critical thinking. Critical thinking goes beyond the skills of logical analysis, since it implies to question the assumptions underlying the habitual ways of thinking and acting and, based on this critical questioning, be prepared to think and do differently.

Planning and time management. This skill involves being able to organize and distribute correctly the time available and distribute it according to the activities necessary to achieve the objectives in the short, medium and long term.

\section{STICKY NOTES TOOLS FOR TEACHERS AND STUDENTS}

A set of free sticky notes tools have been collected for both teachers and students to support them in the teaching and learning tasks in the context of decision-making and in the teamwork's practical works. Next, 11 tools are listed (in alphabetical order) and a brief description follows each of them.

Corkboard [2] allows collaborating, co-creating, collecting and sharing ideas. It is easy to use, through creating a corkboard, then, name the project, add a short description or notes, and upload photos that inspire the idea of Corkboard creation. Start with the initial ideas and create a posting. After that, you can share Corkboard (e.g. you can notify through Facebook or you can collaborate via email). Users can comment the post and invite more people to collaborate. Corkboard gets users notified of new comments on your postings. Moreover, users can post online, via SMS, email or IPhone App and post locations, notes, websites, images, links, etc.

Hott Notes [3] is a free sticky notes reminder for the desktop. A simple, easy-to-use sticky notes software for Windows. Hott notes allows to create checklists to keep track of what you have done and what you have left to do. Hott notes have a simple, yet pleasant interface. This software allows user to choose any color, font, or translucency level for their notes andalso, create and use themes. Editing notes is easy and non-obstructive, it could be done just double-clicking the note body, editing it, and hitting "Done" when the editing process is finished. Hott notes enables managing a large number of notes. A Note Manager allows users view the desktop, wastebasket, and archived notes and perform batch operations on them. 
Linoit [4] is a tool that allows sticking and sharing notes, posts, links, photos, etc. Linoit requires a web browser that contains a canvas service for the sticking tasks. Linoit allows taking a note right away wherever the user is; accessing from users' home, office, or even on the road and post a sticky notes online whenever it is needed; organising the stickies visually, so that users can move the stickies around and change their colors whenever and however they like; having a free layout of pictures and movies, thus, users can position picture archives and movies as they like and share them with other students; sending an Email and take a note online; creating an Email message containing the note and send it to linoit; setting due dates on your stickies, and receive an Email reminder; sharing files among colleagues, attach files to stickies, and share them with colleagues at home and office; using Linoit as a bulletin board among students, creating a group with colleagues to share a canvas and stickies with them; using as a handy tool for seminars and theoretical classes; sharing ideas with texts and pictures, and having a discussion with students; using from smartphones, take notes, post pictures and videos.

Listhings [5] is a tool, that was launched in April 2009, for online sticky notes. It is a free web app for taking notes and all notes are automatically saved to Listhings database servers. Users can access their notes from anywhere and share them with anyone to collaborate in real-time. Currently, this tool has over 60.000 users.

Notepad2 [6] is a fast and light-weight notepad-like text editor with syntax highlighting. This program can be run out of the box without installation, and does not touch the system's registry. It has been created through the Scintilla source code editing component, a free source code editing component for Win32, GTK+, and OS X.

Padlet [7] gives users a blank page (a wall) and they put anything they want on it, anywhere. Padlet is an easy site for students to use. Pose questions, get their answers, and give them feedback - all on the same page. Padlet allows user management and access control, teachers can add and remove people from class. Teachers can revoke access to student graduates while still keeping their data. If new students join, teachers can give them, through Padlet, automatic access to lessons and plans and other stuff that has been created. All padlets, by default, are only visible to the people in the class. Moreover, teachers can also remove all social features like Facebook sharing from padlets. All this without compromising on the ease of sharing with others, including sharing outside the school when users have to. Padlet offers extra security, by limiting access to its content to the walls of its campus. Padlet servers are well protected; using SSL to transfer data between the users' computers and Padlet's servers, the same technology used by banks. Moreover, Padlet backups the data every day. Moreover, Padlet allows branding. Instead of meaningless "padlet", users can customise the wall with school name and logo, subject, etc. Users can get their own subdomain, e.g., mybeautifulschool.padlet.org or even use their own domain mybeautifulschool.com. Padlet allows monitoring tasks, keeping tabs on what is going on - what is being created, what is being shared.. Finally, bigger file uploads are allowed with Padlet, e.g. post a 100MB video, a 50MB photoshop file, and upload files up to $250 \mathrm{MB}$ in size.

Scrumblr [8] is a web-based simulation of a physical agile sprint board that supports real-time collaboration. A template is generated in the web browser, making easy the start of creating a canvas with post-its and sticky notes.

Simple Sticky Notes [9] provides a simple, easy-to-use, free, fast and efficient note taking software. Students can jot down important pieces of information quickly and easily right on their desktop. Simple Sticky Notes lets students to easily create sticky notes and neatly organise them in their desktop. Students can change the note colour and size; create and organize multiple notes and set an alarm to get a note reminder; adjust each note's transparency; stick the note to be always on top; and Hide or display all notes on their desktop from the system tray icon.

Spaaze [10] offers its users so called boards. Each board is an infinite space which resembles a virtual cork. On these boards, items can be put. Currently Spaaze offers 7 different items: Labels, Notes, Bookmarks, Images, Files, Videos and HTML. These items can be edited in place, moved via drag and drop and positioned freely on the board. Therefore, it is possible to arrange a bunch of items any way users like, which makes it possible to use it for many different use cases.

Stickies [11] is a PC utility, just like a real sticky piece of paper. Once on screen, stickies remain where placed until closed, even if reboots. Stickies appearance can be customised; fonts, colours and buttons may be changed, and styles saved. Stickies can be resized and store text or images. Stickies can snap to each other and to the sides of the screen to keep them neatly lined up. Stickies can be attached to a web site, document or folder so they only show when it is on screen. Stickies can be 
transferred from one machine to another either over a TCP/IP network connection, or by using an SMTP mail server or MAPI client. Stickies can be hidden for a certain period, until a specified date and time, or to wake every day, week or month, to act as reminders. Stickies can have alarms set to ensure you notice them at a point you choose. International language, Unicode and RTL text support. Stickies works with Windows XP, Vista, Windows 7 and Windows 8. Stickies is small and simple, it writes to a single text file, and does not alter the registry. AD network administrators can use Group Policy to control settings. API to allow integration with other applications

StickyPad [12] is an easy-to-use tool that lets users place sticky notes on their desktop. Users can type notes to themselves, set meeting and appointment reminders, keep track of a to-do list, and anything else users would do with paper notes. The notes can be printed, pasted into other applications, or emailed to coworkers. Users can even make notes translucent and have them float above other windows, so users can see both the note and what they are working on. When users log off, StickyPad remembers all of their notes, and when they log back on, their notes are still there.

According to [13], and to the best of our knowledge, Linoit [4] and Padlet [7] are two interesting online sticky notes tools, due to the fact that they are web-based and work well on iPads, PCs, Macs, smartphones and tablets. With these two tools, canvas can be managed by students without logins, making them easy to infuse into lessons. Moreover, the post-its are not limited to text, and they can have images, videos, and hyperlinks.

\subsection{Comparing Linoit and Padlet Sticky notes tools}

Communication, collection and collaboration are key factors that support the decision-making process. In this regard, stick notes will allow gathering ideas amongst all the students, aiding the process of decision-making. This is what Linoit [4] and Padlet [7] do, and they do it digitally, with less mess and more interactivity. Linoit [4] and Padlet [7] are quite similar; nevertheless, some differences can be appreciated in the following Table 1.

Table 1. Linoit vs Padlet sticky notes tools (extracted from [13]).

\begin{tabular}{l|l}
\hline \multicolumn{1}{c|}{ Linoit [4] } & \multicolumn{1}{c}{ Padlet [7] } \\
\hline $\begin{array}{l}\text { Does not have a stream layout, but its posts are easier } \\
\text { to drag and sort. }\end{array}$ & $\begin{array}{l}\text { Has a stream layout where posts are placed one below } \\
\text { the other }\end{array}$ \\
\hline
\end{tabular}

Change the colour of the notes and the text. When Does not have support colouring feature for notes and brainstorming what technology could be used for a project, the notes could be colour coded according to what device is required.

Rotate stickies.

Allow contributors to edit and move their own notes

Each time a canvas is loaded on the iPad, the app must be downloaded. Each time users go to or refresh a Lino canvas from iOS or Android, users have to click the Close link.

To post a video, users have to upload it on YouTube, Vimeo, or Ustream first.

Stickies do not appear until the note is completed. text.

Does not have the feature of moderating posts.

Allow contributors to edit and move their own notes

Has a specific URL

Allows the upload of videos

Stickies appear for everyone on Padlet moments after a user double-clicks the background.

Has the option for moderated posts. With moderation turned on, each posts requires the teacher's approval before appearing on the students' screens. Moderating is as simple as clicking Remove or Approve.

Use own background to directly export and upload to Lino (chart, graphic organizer, images from the web or make your own, a PowerPoint slide, etc.)

Does not support printing.

Allows uploading own wallpaper. But it is hard to upload certain files of the background.

Post are in the same position.

Print button under the Share menu. Save posts from in a PDF or Excel file.

Post are not in the same position on the wallpaper on different screens. 


\section{A TOOL TO SUPPORT THE COLLABORATIVE DECISION-MAKING AND PROMOTE TEAMWORK PRACTICES IN DEGREE STUDENTS}

As a result of the comparative analysis performed in section 2 , and according to the needs required in a tool to support students, as future industrial engineers, in the decision-making process through teamwork, when establishing of strategic policies, carrying out brainstorming activities, and creating solutions, the authors have decided to focus on Linoit [4] tool.

Linoit allows organising sticky notes visually, and move them around the canvas. Sticky notes can change their colours for classifying the groups participating in the collaborative decision-making process. The sticky notes can position anywhere in the Canvas (e.g. distributed by groups) and share them with the members of the group and with other members' groups. With Linoit, students can attach files, videos, web-links to the sticky notes and share them with other students. Moreover, students will be able to create an Email message containing notes and send it to Linoit, where it will be posted as a post-it. Linoit will allow the students to share their ideas and to have discussions with colleagues; supporting the creation process, and the decision-making process, from a collaborative perspective.

Setting up a wall in Linoit:

1 The student/teacher needs a device such as a PC, Mac, iPad, iPhone, Android.

2 Create an account. Whenever the user login, the user will be able to access all of the walls he/she ever created under that account.

3 Create a New Canvas. Give it a name and choose its settings. Linoit can choose from the backgrounds Lino provides, or, users can upload their own image to use as the background.

4 Decide how public the canvas will be. It can be just for users' own use, they can let others see the canvas but not add to it, or, they can make it so everyone can see it and contribute to it.

5 If everyone may post stickies, users should be sure to leave "Allow Guests to Post Stickies" checkmarked. That way students will not need a Lino account in order to post.

6 Visit a wall by going to a specific web address in their web browser.

7 On a Lino canvas, a student drags a note from the upper-right corner to begin a post.

8 Digital sticky notes do not have to be limited to text. In addition to being text or a video, a note can have a hyperlink.

9 Users can also attach other files, like images, PDFs, and Word documents, as a way for students to turn in their work or to share it. Or as a way for the teacher to distribute files.

10 Users can save audios recorded online. The tool provides a URL, where the audios are stored. Students simply copy that URL and paste it into a new sticky note.

\section{PRACTICAL APPLICATIONS}

In this section, practical applications with the proposed tool (Linoit), to support the collaborative decision-making and promote teamwork practices, are described; for its use in practical session with students belonging to the Industrial Engineering degree. Linoit will be considered as a tool to aid the decision-making process through the definition of the following practical sessions; (i) the formulation of strategies in an enterprise: using Linoit for the SWOT analysis (Strengths, Weaknesses, Opportunities and Threats) which can be used effectively to build organization strategies; (ii) design thinking: Linoit will support the design thinking methodology, enabling to generate innovative ideas that focus its effectiveness on understanding and solving real problems; and (iii) brainstorming, as a group technique to generate original ideas in a relaxed atmosphere, Linoit will be used as a teamwork tool that facilitates the emergence of new ideas on a subject or problem.

\subsection{Industrial Engineering Degree: Subjects and Practical Sessions}

Industrial Engineering Degree trains graduates with a solid scientific and technological basis that allows them to manage, advise, operate and improve organizations, production and information systems, processes, and services to promote the competitive advantage of companies, taking into account technological and human aspects, and the economic viability of proposals designed. All this, in harmony with professional and business organizations and in accordance with the professional duties recognized by the current law. 
The general objectives of the Industrial Engineering Degree are among others, the following ones: (i) take decisions and reason critically, (ii) develop creativity, (iii) solve problems with initiative and entrepreneurship, (iv) implement the principles and methods of high quality, (v) work in a multilingual and multidisciplinary environment, (vi) manage information from various sources and, where appropriate, computer search tools and classification of library resources or mono or multimedia information, (vii) understand the ethical and professional liability of professional activity, and (viii) learn stand-alone with the belief that learning is a continuum in life.

The subjects included in the Industrial Engineering degree are devoted to deal with the aforementioned general objectives. In the data sheet [20], which describes the Industrial Engineering degree, it is shown the set of subjects that conforms it. Amongst these subjects, the paper focuses its attention in those in which the decision-making process is addressed, such as the Quantitative Methods For Industrial Organisation, Strategic Management, etc. Going deeper, the decision-making process is treated in this paper from the practical perspective. In this regard, a set of three practical sessions are proposed to deal with the decision-making, using Lino [4] sticky note tool.

\subsection{SWOT analysis}

SWOT analysis (alternatively SWOT matrix) is an acronym for strengths, weaknesses, opportunities, and threats-and it is a structured planning method that evaluates those four elements of a project or business venture. A SWOT analysis can be carried out for a company, product, place, industry, or person. It involves specifying the objective of the business venture or project and identifying the internal and external factors that are favorable and unfavorable to achieve that objective. Some authors credit SWOT to Albert Humphrey, who led a convention at the Stanford Research Institute (now SRI International) in the 1960s and 1970s using data from Fortune 500 companies[1][2]. However, Humphrey himself did not claim the creation of SWOT, and the origins remain obscure. The degree to which the internal environment of the firm matches with the external environment is expressed by the concept of strategic fit. The four elements of a SWOT analysis are: (i) Strengths: characteristics of the business or project that give it an advantage over others; (ii) Weaknesses: characteristics that place the business or project at a disadvantage relative to others; (iii). Opportunities: elements that the business or project could exploit to its advantage and (iv) Threats: elements in the environment that could cause trouble for the business or project.

Identification of SWOTs is important because they can inform later steps in planning to achieve the objective. First, decision makers should consider whether the objective is attainable, given the SWOTs. If the objective is not attainable, they must select a different objective and repeat the process.

Users of SWOT analysis must ask and answer questions that generate meaningful information for each category (strengths, weaknesses, opportunities, and threats) to make the analysis useful and find their competitive advantage.

An application of the SWOT analysis can be seen in Figure 1 and through the following URL: http://linoit.com/users/beaanna26/canvases/SWOTAnalysis.

\subsection{Design Thinking}

It is widely recognized that design is a key activity of engineering [14] since the students of Industrial Engineering degree should have skills to design effective solutions to meet social and business needs. The purpose of engineering education is to graduate engineers who can design, and that design thinking is complex [15].

Brown [16] states that the best way to describe the process of design is metaphorically as a system of spaces rather than a predetermined set of ordered steps. The spaces demarcate different types of activities related that in conjunction form the continuum of innovation. Design thinking seems to be a chaotic process for those who are not used with it. However, over the life cycle of a project, participants in such a project come to see that the design thinking process has sense, although its disordered architecture, and achieve the expected results, even though its development is very different from the processes of other type of business activities.

According to the system of spaces perspective defined by [16], the author explains that the design thinking process of a project consists of three spaces: (i) inspiration: for the circumstances (it can be a problem, an opportunity or both), that motivate the search for solutions; (ii) ideation: that covers the generation, development and test of the ideas in order to develop real solutions and (iii) 
implementation: to define the exploitation activities to reach the market. Projects will go iteratively through this clycle the needed times till the solution and the path to markeyt has been achived.

In order to develop the necessary skills among the future Engineers to promote the design thinking, a practical session, where students have to work on new product development, has been defined. The session consists of developing a new product with predefined requirements and other unrestricted aspects to be decided in order to promote the inspiration and ideation stages of the decision thinking process. To do so, Linoit is a very powerful tool to guide students in the disordered process of design thinking in order to register the main ideas and concepts and to give a little bit of order and direction to this process.

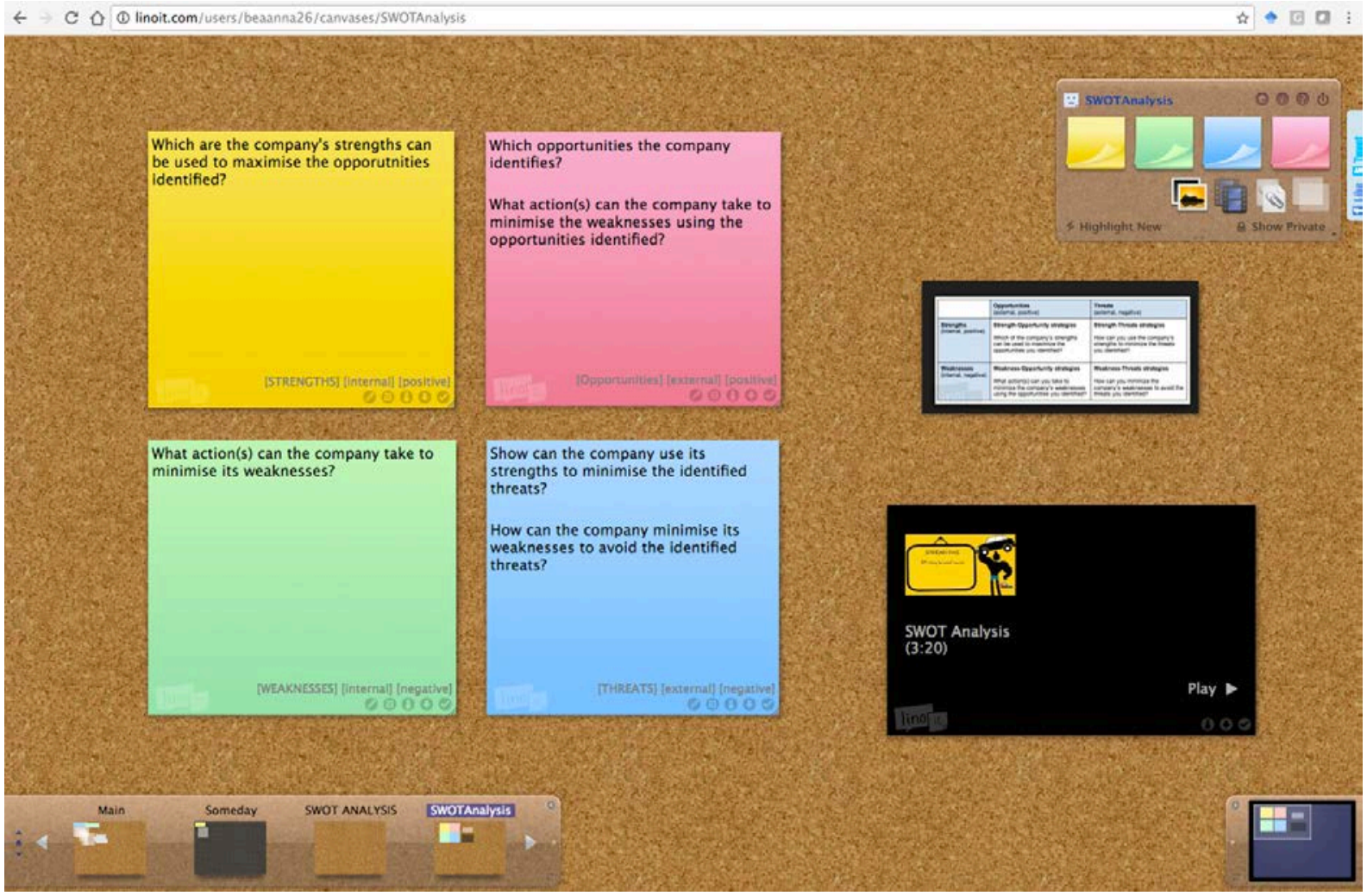

Figure 1. SWOT analysis: Linoit tool (http://linoit.com/users/beaanna26/canvases/SWOTAnalysis).

\subsection{Brainstorming}

Since the publication of Osborn's [17] book: "Applied Imagination", group brainstorming is considered as a very effective technique for the generation of original ideas. As suggested by [19], Osborn's [17] book highlights four main principles for the brainstorming process:

- Criticism is ruled out. Adverse judgment of ideas should be postponed since the main objective of the brainstorming process is the generation of varied and diverse options.

- Freewheeling is welcome. The wilder the idea, the better. As hostile opinions are postponed, it is really wanted the generation of wild and unusual ideas.

- Quantity is wanted. The greater the number of ideas, the greater the likelihood of useful ideas.

- Combination and improvement are sought. The collaboration among the participant should be addressed to the enhancement of the ideas generated and the generation of new ones.

In the past, the brainstorming research has focused almost completely on productivity (i.e., number of ideas generated) since there are trends that assure that brainstorming groups generate fewer ideas, than individuals [18]. Currently, the research lines are focused on the electronic brainstorming

However in the proposed practical session, the main objective pursued is not focused on the productivity of the brainstorming group indeed but in the teamwork skills, that is how the students of the group interact each other, produce original ideas and how the collaboration among them, makes 
the ideas improve and even though generate new ones. The practical session consists of the definition of an engineering problem, e.g. loss of products' traceability within the storage area of an enterprise and the students, using the brainstorming technique, have to analyse the causes of such a problem and propose ideas to solve it. For the generation of the ideas, Linoit supports the visualization of the proposals and aids the participants to improve the suggestions proposed. The practical session consists of 5 main steps:

- Preparation of the session. This step is mainly focused on the creation of a confortable environment for encouraging the generation of ideas. In this preparatory phase, it is established the number of students per group and also the appointment of a facilitator, whose main task is to point direction to the group.

- Discussing the topic. The teacher explains to the group why the session is necessary, the main problem, characteristics and some general background information about the topic. Moreover, it is explained that the tool to gather all the ideas generated is Linoit and gives some general guidelines about how this tool works.

- Establishing the rules. The teacher sets the rules for the session, highlighting the four main principles of the brainstorming technique defined by [17] and the schedule for the session.

- Running the session. In this stage, the students are generating their ideas and the facilitator is registering such ideas through the Linoit tool as an efficient source to easily visualize the proposals made by the group.

- Organizing ideas. Organizing ideas prepares the group for the evaluation and discussion of the ideas generated in order to refine them or generate new ones. The final objective of this stage is to achieve a consensus about possible methods to solve the problem. In this stage, Linoit tool has a very important role as it is key to display in an organized manner the ideas generate to facilitate the ideas organization process. Moreover, the prioritization of the ideas is also supported by Linoit through for example using different colours.

\section{CONCLUSIONS}

Conclusions are provided, considering the utility and the usefulness of the proposed tool, Linoit, for the decision-making process in teamworks, in different areas of Industrial Engineering. To this regard, students of the Industrial Engineering degree will take advantage of an online and open-source tool for working in groups (teamwork) and dealing with relevant issues, such as strategies formulation, design thinking and brainstorming.

The SWOT analysis is a very complex task that manages a lot of information. Linoit helps students with the information sharing and management. Putting in order ideas, and graphically showing the different views of the strategies creation and management. With regard to the design thinking, Linoit is a very useful tool as it provides order to a disordered process such as the design thinking. It facilitates the visualization of the proposals generated during the design process. The advantages of using Linoit in brainstorming sessions is related to the support that the tool offers in order to organize the ideas, prioritize them (with for example different colours) to facilitate the improvement of such ideas or even though, the generation of new ones.

\section{REFERENCES}

[1] https://elearningindustry.com/free-sticky-notes-tools-for-teachers-students

[2] http://www.corkboard.it/?utm_campaign=elearningindustry.com\&utm_source=/free-sticky-notestools-for-teachers-students\&utm_medium=link

[3] http://www.hottnotes.com/

[4] http://en.linoit.com/

[5] http://listhings.com/canvas/l7umCbU

[6] http://www.flos-

freeware.ch/notepad2.html?utm_campaign=elearningindustry.com\&utm_source=/free-stickynotes-tools-for-teachers-students\&utm_medium=link 
[7] https://padlet.com/?utm_campaign=elearningindustry.com\&utm_source=/free-sticky-notestools-for-teachers-students\&utm_medium=link

[8] http://scrumblr.ca/?utm_campaign=elearningindustry.com\&utm_source=/free-sticky-notes-toolsfor-teachers-students\&utm_medium=link

[9] http://www.simplestickynotes.com/help/

[10] http://www.spaaze.com/about

[11] http://www.zhornsoftware.co.uk/stickies/?utm_campaign=elearningindustry.com\&utm_source=/f ree-sticky-notes-tools-for-teachers-students\&utm_medium=link

[12] http://www.greeneclipse.com/stickypad.html?utm_campaign=elearningindustry.com\&utm_sourc e=/free-sticky-notes-tools-for-teachers-students\&utm_medium=link

[13] http://learninginhand.com/blog/2013/6/2/communicate-collect-collaborate-with-sticky-notes

[14] Simon, H. A. (1996). The sciences of the artificial. MIT press.

[15] Dym, C. L., Agogino, A. M., Eris, O., Frey, D. D., \& Leifer, L. J. (2005). Engineering design thinking, teaching, and learning. Journal of Engineering Education, 94(1), 103-120.

[16] Brown, T. (2008). Design thinking. Harvard business review, 86(6), 84.

[17] Osborn, A. F. (1953). Applied imagination. Oxford, UK: Charles Scribner's Sons.

[18] Rietzschel, E. F., Nijstad, B. A., \& Stroebe, W. (2006). Productivity is not enough: A comparison of interactive and nominal brainstorming groups on idea generation and selection. Journal of Experimental Social Psychology, 42(2), 244-251.

[19] Lamm, H., \& Trommsdorff, G. (1973). Group versus individual performance on tasks requiring ideational proficiency (brainstorming): A review. European journal of social psychology, 3(4), 361-388.

[20] http://www.upv.es/titulaciones/GIOI/menu_920724i.html 